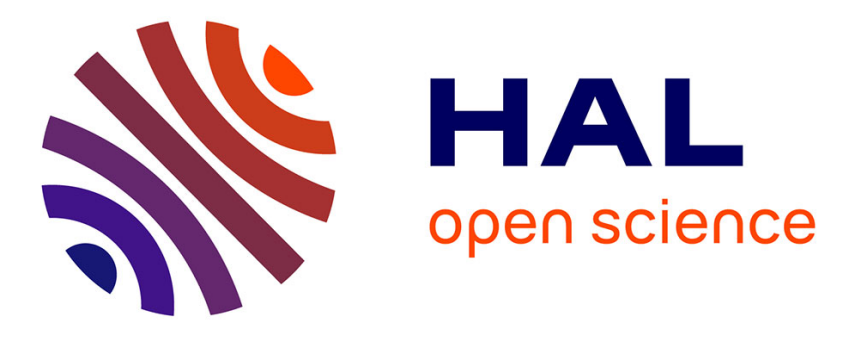

\title{
Palindromic Discontinuous Galerkin Method
}

David Coulette, Emmanuel Franck, Philippe Helluy, Michel Mehrenberger,

\author{
Laurent Navoret
}

\section{To cite this version:}

David Coulette, Emmanuel Franck, Philippe Helluy, Michel Mehrenberger, Laurent Navoret. Palindromic Discontinuous Galerkin Method. Finite volumes for complex applications VIII-hyperbolic, elliptic and parabolic problems, Jun 2017, Lille, France. pp.6, 10.1007/978-3-319-57394-6_19 . hal01653049

\section{HAL Id: hal-01653049 \\ https://hal.science/hal-01653049}

Submitted on 1 Dec 2017

HAL is a multi-disciplinary open access archive for the deposit and dissemination of scientific research documents, whether they are published or not. The documents may come from teaching and research institutions in France or abroad, or from public or private research centers.
L'archive ouverte pluridisciplinaire HAL, est destinée au dépôt et à la diffusion de documents scientifiques de niveau recherche, publiés ou non, émanant des établissements d'enseignement et de recherche français ou étrangers, des laboratoires publics ou privés. 


\title{
PALINDROMIC DISCONTINUOUS GALERKIN METHOD
}

\author{
DAVID COULETTE, EMMANUEL FRANCK, PHILIPPE HELLUY, MICHEL \\ MEHRENBERGER, LAURENT NAVORET
}

\begin{abstract}
We present a high-order scheme for approximating kinetic equations with stiff relaxation. The construction is based on a high-order, implicit, upwind Discontinuous Galerkin formulation of the transport equations. In practice, because of the triangular structure of the implicit system, the computations are explicit. High order in time is achieved thanks to a palindromic composition method. The whole method is asymptotic-preserving with respect to the stiff relaxation and remains stable even with large CFL numbers.
\end{abstract}

\section{INTRODUCTION}

The Lattice Boltzmann Method (LBM) is a general method for solving systems of conservation laws [4]. The LBM relies on a kinetic representation of the system of conservation laws by a small set of transport equations coupled through a stiff relaxation source term. The kinetic model is solved with a splitting method, in which the transport and relaxation steps are treated separately. Usually, the transport is exactly solved by the characteristic method.

The main drawback of the LBM is that it requires regular grids and that the time step $\Delta t$ is imposed by the grid step $\Delta x$. In this paper, we thus prefer to solve the transport equation with a Discontinuous Galerkin (DG) method. We extend the DGLBM of [11] in several directions. The first improvement is to apply an implicit DG method instead of an explicit one for solving the transport equations. This can be done at almost no additional cost. Indeed, with an upwind numerical flux, the linear system of the implicit DG method is triangular and, in the end, can be solved explicitly. In this way, we obtain stable methods even with high CFL numbers. This kind of ideas can be found for instance in $[2,5]$.

The second improvement is to construct a high order time integrator that remains accurate even for infinitely fast relaxation, thanks to a composition method [10].

We validate our approach on a few one-dimensional test cases.

\section{A VECTORIAL KINETIC MODEL}

We consider the following kinetic equation

$$
\partial_{t} \mathbf{f}+\sum_{k=1}^{d} \mathbf{V}^{k} \partial_{k} \mathbf{f}=\frac{1}{\tau}\left(\mathbf{f}^{e q}(\mathbf{f})-\mathbf{f}\right)
$$

The unknown is a vectorial distribution function $\mathbf{f}(\mathbf{x}, t) \in \mathbb{R}^{n}$ depending on the space variable $\mathbf{x} \in \mathbb{R}^{D}$ and time $t \in \mathbb{R}$. The relaxation time $\tau$ is a small positive constant. The constant matrices $\mathbf{V}^{k}, 1 \leq k \leq d$ are diagonal. In other words (2.1)

Key words and phrases. Lattice Boltzmann; Discontinuous Galerkin; implicit; composition method; high order, stiff relaxation. 
is a set of transport equations at constant velocities coupled through a stiff BGK relaxation.

Generally this kinetic model is an approximation of an underlying hyperbolic system of conservation laws. The macroscopic conservative variables $\mathbf{w}(\mathbf{x}, t) \in \mathbb{R}^{m}$ are obtained through a linear transformation

$$
\mathbf{w}=\mathbf{P f}
$$

where $\mathbf{P}$ is a $m \times n$ matrix. Generally the number of conservative variables is smaller than the number of kinetic data: $m<n$. The equilibrium distribution $\mathbf{f}^{e q}(\mathbf{f})$ is such that

$$
\mathbf{P f}=\mathbf{P f}^{e q}(\mathbf{f})
$$

and

$$
\mathbf{w}=\mathbf{P f}=\mathbf{P g} \Rightarrow \mathbf{f}^{e q}(\mathbf{f})=\mathbf{f}^{e q}(\mathbf{g}) .
$$

When $\tau \rightarrow 0$, the kinetic equations provide an approximation of the system of conservation laws

$$
\partial_{t} \mathbf{w}+\sum_{k=1}^{D} \partial_{k} \mathbf{q}^{k}(\mathbf{w})=0,
$$

where the flux is given by

$$
\mathbf{q}^{k}(\mathbf{w})=\mathbf{P} \mathbf{V}^{k} \mathbf{f}^{e q}(\mathbf{f})
$$

The flux is indeed a function of $\mathbf{w}$ only because of (2.2). For more details, we refer to $[3,1,7]$.

In the following, without loss of generality, we shall only consider the onedimensional case $D=1$.

\section{Implicit Discontinous Galerkin (DG) Method}

In this section, we briefly recall how to approximate the transport equation by a DG method of order $d$, based on Lagrange polynomials. We wish to approximate the solution $f(x, t)$ of a scalar transport equation in the case $v>0$ (the case $v<0$ is obtained in a similar way)

$$
\partial_{t} f+v \partial_{x} f=0
$$

The space variable $x \in[a, b]$. We split the interval $[a, b]$ into $N_{x}$ cells of size $h=(b-a) / N_{x}$. In each cell $C_{\ell}, \ell=0 \ldots N_{x}-1$, we consider the $d+1$ GaussLobatto points $x_{\ell, i}, i=0 \ldots d$, associated to quadrature weights $\omega_{\ell, i}$. The DG basis function $\varphi_{\ell, i}$ has its support in cell $\ell$ and in addition satisfies the interpolation property $\varphi_{\ell, i}\left(x_{\ell, j}\right)=\delta_{i j}$. The transported function $f(x, t)$ is then approximated by an expansion on the DG basis

$$
f(x, t) \simeq f_{h}(x, t)=\sum_{j=0}^{d} f_{\ell, j}(t) \varphi_{\ell, j}(x), \quad x \in C_{\ell} .
$$

We can also identify $f_{h}$ with a vector of $\mathbb{R}^{N}, N=N_{x}(d+1)+2, \mathbf{f}_{h}=\left(f_{0}(t), f_{0,0}(t), f_{0,1}(t), \ldots, f_{N_{x}-1, d}(t), f_{N-1}(t)\right)$. It is useful to consider the boundary values $f_{0}=f_{-1, d}$ and $f_{N_{x}, 0}=f_{N-1}$ as artificial unknowns. For simplicity, we will also assume that the boundary conditions do not depend on time. Now we apply the DG formulation to $f_{h}$ : for all cell $C_{\ell}$ and all test function $\varphi_{\ell, i}$

$$
\int_{C_{\ell}}\left(\partial_{t} f_{h}+v \partial_{x} f_{h}\right) \varphi_{\ell, i}+v\left(f_{\ell, 0}-f_{\ell-1, d}\right) \varphi_{\ell, i}\left(x_{\ell, 0}\right)=0 .
$$


Let us point out the upwind nature of the formulation (3.1): when $v>0$, for computing the values inside cell $C_{\ell}$ we only need the knowledge of the values inside cell $C_{\ell-1}$, or the left boundary condition. Therefore, after applying a Gauss-Lobatto quadrature to (3.1), we obtain a set of linear differential equations

$$
\partial_{t} \mathbf{f}_{h}+\mathbf{L}_{h} \mathbf{f}_{h}=0
$$

where $\mathbf{L}_{h}$ is a lower block-triangular matrix (with a good numbering of the unknowns). The diagonal blocks are of size $(d+1) \times(d+1)$. If the velocity $v<0$, the structure is similar. Therefore, in the following, we adopt the same notation $\mathbf{L}_{h}$ for the scalar or vectorial DG transport operator.

\section{High order time integration}

We can also define an approximation $\mathbf{N}_{h}$ of the collision operator $\mathbf{N}$ defined by $\mathbf{N f}=\left(\mathbf{f}^{e q}(\mathbf{f})-\mathbf{f}\right) / \tau$. The DGLBM approximation of (2.1) finally reads

$$
\partial_{t} \mathbf{f}_{h}=\mathbf{L}_{h} \mathbf{f}_{h}+\mathbf{N}_{h} \mathbf{f}_{h} .
$$

We use the following Crank-Nicolson second order time integrator for the transport equation:

$$
\exp \left(\Delta t \mathbf{L}_{h}\right) \simeq T_{2}(\Delta t):=\left(\mathbf{I}+\frac{\Delta t}{2} \mathbf{L}_{h}\right)\left(\mathbf{I}-\frac{\Delta t}{2} \mathbf{L}_{h}\right)^{-1} .
$$

Similarly, for the collision integrator, we use

$$
\exp \left(\Delta t \mathbf{N}_{h}\right) \mathbf{f}_{h} \simeq C_{2}(\Delta t) \mathbf{f}_{h}=\frac{(2 \tau-\Delta t) \mathbf{f}_{h}}{2 \tau+\Delta t}+\frac{2 \Delta t \mathbf{f}_{h}^{e q}\left(\mathbf{f}_{h}\right)}{2 \tau+\Delta t} .
$$

When $\tau \rightarrow 0$, it becomes

$$
C_{2}(\Delta t) \mathbf{f}_{h}=2 \mathbf{f}_{h}^{e q}\left(\mathbf{f}_{h}\right)-\mathbf{f}_{h} .
$$

An integrator $M_{2}(\Delta t)$ is time-symmetric if it satisfies

$$
M_{2}(-\Delta t)=M_{2}(\Delta t)^{-1}, \quad M_{2}(0)=I d .
$$

This property implies that $M_{2}$ is necessarily a second order approximation of the exact integrator $[10,8]$. As explained in [6], $T_{2}$ and $C_{2}$ are time-symmetric when $\tau>0$. But, because of (4.3), $C_{2}$ is no more symmetric for $\tau=0$. Therefore, the usual Strang splitting operator is not time-symmetric either. We rather consider the following splitting method, which is time-symmetric and remains second order accurate even for infinitely fast relaxation:

$$
M_{2}(\Delta t)=T_{2}\left(\frac{\Delta t}{4}\right) C_{2}\left(\frac{\Delta t}{2}\right) T_{2}\left(\frac{\Delta t}{2}\right) C_{2}\left(\frac{\Delta t}{2}\right) T_{2}\left(\frac{\Delta t}{4}\right) .
$$

By palindromic compositions of the second order method $M_{2}$ it is then very easy to achieve any even order of accuracy. See $[10,8,6]$. A general palindromic scheme with $s+1$ steps has the form

$$
M_{p}(\Delta t)=M_{2}\left(\gamma_{0} \Delta t\right) M_{2}\left(\gamma_{1} \Delta t\right) \cdots M_{2}\left(\gamma_{s} \Delta t\right),
$$

where the $\gamma_{i}$ 's are real numbers such that

$$
\gamma_{i}=\gamma_{s-i}, \quad 0 \leq i \leq s .
$$

For $p=4$ and $s=4$ we have for example the fourth-order Suzuki scheme (see $[12,8,10])$

$$
\gamma_{0}=\gamma_{1}=\gamma_{3}=\gamma_{4}=\frac{1}{4-4^{1 / 3}}, \quad \gamma_{2}=-\frac{4^{1 / 3}}{4-4^{1 / 3}} .
$$


This scheme requires five stages and one negative time step. For $p=6$ and $s=8$, we have also the sixth-order Kahan-Li scheme [9] given by:

$$
\begin{array}{cc}
\gamma_{0}=\gamma_{8}= & 0.3921614440073141392756553330038 \ldots \\
\gamma_{1}=\gamma_{7}= & 0.332599136789359438604272125325 \ldots \\
\gamma_{2}=\gamma_{6}= & -0.7062461725576393598098453372227 \ldots \\
\gamma_{3}=\gamma_{5}= & 0.0822135962935508002304427053341 \ldots \\
\gamma_{4}= & 0.798543990934829963398950353048 \ldots
\end{array}
$$

The methods (4.6) and (4.7) require to apply the elementary collision or transport bricks $C_{2}$ and $T_{2}$ with negative time steps $-\Delta t<0$.

The exact transport operator is perfectly reversible. If we were using an exact characteristic solver, negative time steps would not cause any problem. However, the DG approximation introduces a slight dissipation due to upwinding. In order to ensure stability, we have thus to replace $T_{2}(-\Delta t)$ with a more stable operator. This can be done by observing that solving $\partial_{t} f+v \partial_{x} f=0$ for negative time $t<0$ is equivalent to solve $\partial_{t^{\prime}} f-v \partial_{x} f=0$ for $t^{\prime}=-t>0$. Therefore we simply apply the DG solver $T_{2}^{\prime}(\Delta t)$ with opposite velocities instead of $T_{2}(-\Delta t)$.

The numerical collision operator $C_{2}$ is reversible when $\tau \rightarrow 0$. Actually, it does not depend on $\Delta t$ anymore (see (4.3)). In this stage, negative time steps do not cause any difficulty, at least when $\tau \ll \Delta t$.

\section{Numerical RESUlts}

In this section we consider an isothermal compressible flow of density $\rho$ and velocity $u$. The sound speed is fixed to $c=0.6$. The conservative system is given by $m=2$ and $w=(\rho, \rho u), q(w)=\left(\rho u, \rho u^{2}+c^{2} \rho\right)$. The kinetic model is given by $n=4$ and

$$
\begin{gathered}
\mathbf{V}=\operatorname{diag}(-\lambda, \lambda,-\lambda, \lambda), \quad \mathbf{P}=\left(\begin{array}{cccc}
1 & 1 & 0 & 0 \\
0 & 0 & 1 & 1
\end{array}\right), \\
f_{2 k-1}^{e q}=\frac{w_{k}}{2}-\frac{q(w)_{k}}{2 \lambda}, \quad f_{2 k}^{e q}=\frac{w_{k}}{2}+\frac{q(w)_{k}}{2 \lambda}, \quad k=1,2 .
\end{gathered}
$$

The lattice velocity $\lambda$ has to satisfy the sub-characteristic condition $\lambda>|u|+c$.

5.1. Smooth solution. For the first validation of the method we consider a test case with a smooth solution, in the fluid limit $\tau=0$. The initial condition is given by

$$
\rho(x, 0)=1+e^{-30 x^{2}}, \quad u(x, 0)=0 .
$$

The sound speed is set to $c=0.6$ and the lattice velocity to $\lambda=2$. We define the CFL number $\beta=\lambda \Delta t / \delta$, where $\delta$ be the minimal distance between two GaussLobatto points in the mesh. First, the CFL number is fixed to $\beta=5$. We consider a sufficiently large computational domain $[a, b]=[-2,2]$ and a sufficiently short final time $t_{\max }=0.4$ so that the boundary conditions play no role. The reference solution $\mathbf{f}\left(\cdot, t_{\max }\right)$ is computed numerically with a very fine mesh. In the DG solver the polynomial order in $x$ is fixed to $d=5$.

On Figure 5.1 (left picture) we give the results of the convergence study for the smooth solution. The considered error is the $L^{2}$ norm of $\mathbf{f}_{h}\left(\cdot, t_{\max }\right)-\mathbf{f}\left(\cdot, t_{\max }\right)$.

We make the same experiment with $\beta=50$. The convergence study for the Suzuki and Kahan-Li schemes is also presented on Figure 5.1 (right picture). At high CFL, not only the scheme remains stable, but the high accuracy is also preserved. 

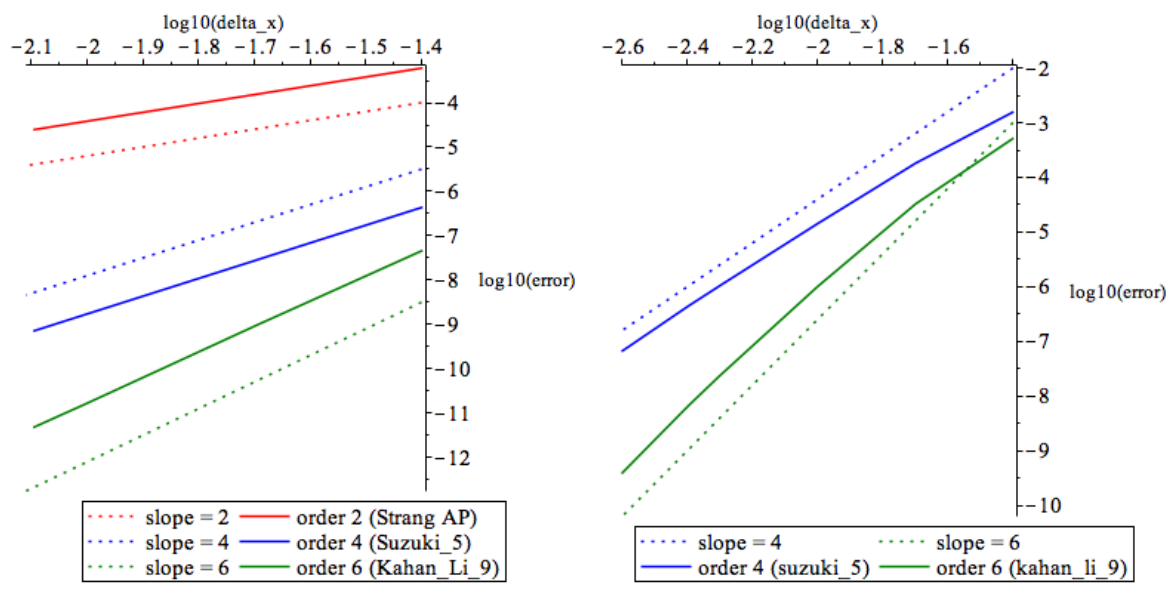

FiguRe 5.1. Convergence study for several palindromic methods, order 2 (red), 4 (blue) and 6 (green). The dotted lines are reference lines with slopes 2,4 and 6 respectively. Left: CFL number $\beta=5$. Right: CFL number $\beta=50$.
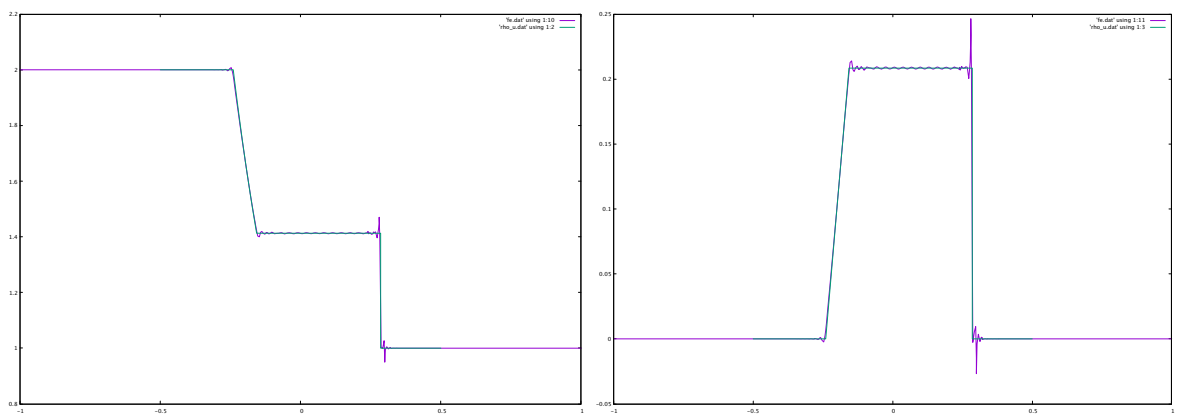

Figure 5.2. Riemann problem with $\tau=0$. Comparison of the exact solution (green curve), and the numerical sixth-order solution (purple curve). Left: density. Right:velocity.

5.2. Behavior for discontinuous solutions. We have also experimented the scheme for discontinuous solutions. Of course, in this case the effective order of the method cannot be higher than one and we expect Gibbs oscillations near the discontinuities. On the interval $[a, b]=[-1,1]$, we consider a Riemann problem with the following initial condition

$$
\rho(x, 0)=\left\{\begin{array}{ll}
2 & \text { if } x<0, \\
1 & \text { otherwise. }
\end{array}, \quad u(x, 0)=0 .\right.
$$

We consider numerical results in the fluid limit $\tau=0$. On Figure 5.2 we compare the sixth-order numerical solution with the exact one at $t=t_{\max }=0.4$ for a CFL number $\beta=3$ and $N_{x}=100$ cells. We observe that the high order scheme is able to capture a precise rarefaction wave and the correct position of the shock wave. We observe oscillations in the shock wave, as expected. 


\section{Conclusion}

In this paper we have described a new numerical method, the Palindromic Discontinuous Galerkin Method, for solving kinetic equations with stiff relaxation. The method has the following features:

- The transport solver is based on an implicit, high-order, upwind Discontinuous Galerkin method. Thanks to the upwind flux, the linear system to be solved at each time step is triangular.

- Time integration is high-order, based on a general palindromic composition method. We have tested it up to order 6 . The method is low-storage.

- The scheme remains stable and accurate at high CFL numbers and for infinitely fast relaxation.

- The method is general, can be extended to higher dimensions, and is well adapted to parallel computers.

We are currently working on the extension of the method to higher dimensions and to optimizations of the implementation on hybrid computers. It is also important in practical applications to extend the method with more general boundary conditions.

\section{REFERENCES}

[1] Aregba-Driollet, D., Natalini, R.: Discrete kinetic schemes for multidimensional systems of conservation laws. SIAM Journal on Numerical Analysis 37(6), 1973-2004 (2000)

[2] Bey, J., Wittum, G.: Downwind numbering: Robust multigrid for convection-diffusion problems. Applied Numerical Mathematics 23(1), 177-192 (1997)

[3] Bouchut, F.: Construction of BGK models with a family of kinetic entropies for a given system of conservation laws. Journal of Statistical Physics 95(1-2), 113-170 (1999)

[4] Chen, S., Doolen, G.D.: Lattice Boltzmann method for fluid flows. Annual Review of Fluid Mechanics 30(1), 329-364 (1998)

[5] Coquel, F., Nguyen, Q.L., Postel, M., Tran, Q.H.: Large time step positivity-preserving method for multiphase flows. In: Hyperbolic Problems: Theory, Numerics, Applications, pp. 849-856. Springer (2008)

[6] Coulette, D., Franck, E., Helluy, P., Mehrenberger, M., Navoret, L.: Palindromic discontinuous Galerkin method for kinetic equations with stiff relaxation. arXiv preprint arXiv:1612.09422 (2016)

[7] Graille, B.: Approximation of mono-dimensional hyperbolic systems: A lattice Boltzmann scheme as a relaxation method. Journal of Computational Physics 266, 74-88 (2014)

[8] Hairer, E., Lubich, C., Wanner, G.: Geometric numerical integration: structure-preserving algorithms for ordinary differential equations, vol. 31. Springer Science \& Business Media (2006)

[9] Kahan, W., Li, R.C.: Composition constants for raising the orders of unconventional schemes for ordinary differential equations. Mathematics of Computation of the American Mathematical Society 66(219), 1089-1099 (1997)

[10] McLachlan, R.I., Quispel, G.R.W.: Splitting methods. Acta Numerica 11, 341-434 (2002)

[11] Shi, X., Lin, J., Yu, Z.: Discontinuous Galerkin spectral element lattice Boltzmann method on triangular element. International Journal for Numerical Methods in Fluids 42(11), 1249-1261 (2003)

[12] Suzuki, M.: Fractal decomposition of exponential operators with applications to many-body theories and Monte Carlo simulations. Physics Letters A 146(6), 319-323 (1990)

Current address: Philippe Helluy, IRMA Strasbourg, Inria Tonus, helluy@unistra.fr 